\title{
Cuidadores de idosos em estratégias de saúde da família: o estresse destes indivíduos
}

\author{
Elderly caregivers in family health strategies: the stress of these individuals
}

Cuidadores de ancianos en estrategias de salud familiar: el estrés de estos individuos

Sandra Maria de Mello Cardoso ${ }^{1 *}$, Andressa Peripolli Rodrigues ${ }^{1}$, Sara Gallert Sperling ${ }^{2}$, Neiva Claudete Brondani Machado', Lucimara Sonaglio Rocha ${ }^{1}$, Margot Agathe Seiffert', Mariéli Terezinha Krampe Machado ${ }^{1}$, Rita Fernanda Monteiro Fernandes ${ }^{1}$.

\section{RESUMO}

Objetivo: Investigar o estresse dos cuidadores de idosos de Estratégias de Saúde da Família da zona rural e da zona urbana. Métodos: Trata-se de uma pesquisa com abordagem qualitativa e descritiva. A amostra foi composta por 10 cuidadores de idosos de quatro estratégias, uma rural e três na área urbana. Para coleta dos dados foram utilizados um questionário e a Escala de Zarit. A análise dos dados foi mediante análise temática de conteúdo. Resultados: Nessa pesquisa constatou-se que cinco cuidadores são familiares, do sexo feminino, três com mais de 60 anos e são esposas, e duas filhas (35 e 38 anos) e que não recebem remuneração pelo trabalho. Na avaliação da sobrecarga dos cuidadores familiares, um dos cuidadores apresentou sobrecarga moderada a severa e os demais sobrecarga moderada. Conclusão: É necessária uma rede de atenção à saúde do idoso para ofertar diversos tipos de serviços, com apoio financeiro e estruturada de forma regionalizada e integrada.

Palavras-chave: Idoso, Cuidadores, Estresse psicológico.

\section{ABSTRACT}

Objective: To investigate the stress of caregivers of elderly Family Health Strategies in rural and urban areas. Methods: It is a research with qualitative and descriptive approach. The sample consisted of 10 elderly caregivers of four strategies, one rural and three in the urban area. For data collection a questionnaire and the Zarit Scale were used. Data analysis was through thematic content analysis. Results: In this research it was found that five caregivers are family members, female, three over 60 years old and are wives, and two daughters (35 and 38 years old) who do not receive remuneration for their work. In the assessment of the burden of family caregivers, one of the caregivers presented moderate to severe overload and the other moderate overload. Conclusion: An elderly health care network is needed to offer various types of services, with financial and structured support in a regionalized and integrated manner.

Key words: Aged, Caregivers, Stress psychological.

\section{RESUMEN}

Objetivo: Investigar el estrés de los cuidadores de ancianos de las Estrategias de Salud Familiar en áreas rurales y urbanas. Métodos: Es una investigación con enfoque cualitativo y descriptivo. La muestra consistió en 10 cuidadores mayores de cuatro estrategias, una rural y tres en el área urbana. Para la recolección de datos se utilizó un cuestionario y la escala de Zarit. El análisis de datos se realizó mediante análisis de

\footnotetext{
${ }^{1}$ Instituto Federal de Educação, Ciência e Tecnologia Farroupilha (IFFar), Santo Ângelo - RS.

*E-mail: sandra.cardoso@iffarroupilha.edu.br

${ }^{2}$ Prefeitura Municipal de Coronel Barros, Coronel Barros - RS.
} 
contenido temático. Resultados: En esta investigación se encontró que cinco cuidadores son miembros de la familia, mujeres, tres mayores de 60 años y son esposas, y dos hijas (35 y 38 años) que no reciben remuneración por su trabajo. En la evaluación de la carga de los cuidadores familiares, uno de los cuidadores presentó sobrecarga moderada a severa y la otra sobrecarga moderada. Conclusión: Se necesita una red de atención médica para personas mayores para ofrecer varios tipos de servicios, con apoyo financiero y estructurado de manera regionalizada e integrada.

Palabras clave: Anciano, Cuidadores, Estrés psicológico.

\section{INTRODUÇÃO}

A transição demográfica está ocorrendo em todo o mundo e o envelhecimento faz parte da realidade da maioria das sociedades. Acompanhando a tendência global, o aumento da longevidade dos brasileiros vem progressivamente aumentando ao longo do tempo. Para o ano de 2018, a expectativa de vida ao nascer, foi de 76,3 anos, significando um incremento de 30,8 anos para ambos os sexos quando comparado ao mesmo indicador observado no ano de 1940 (BRASIL, 2019).

Assim, com o crescimento populacional de idosos, cresce também a necessidade de uma abordagem coletiva de saúde, visando diminuir a supressão das necessidades assistenciais desta população emergente. Desta forma, tal quadro mostra-se como um desafio para os profissionais de saúde e para a sociedade de forma geral, uma vez que, estratégias assistenciais inovadoras devem ser implementadas a fim de buscar uma melhoria da qualidade de vida da população senescente. (ZAMBONI C, et al., 2011).

Muitos idosos continuam trabalhando, fazendo parte da força produtiva e da sociedade de consumo. No entanto, uma parte significativa desse grupo etário tem alguma ou múltiplas dependências físicas, mentais e/ou sociais. O aumento das doenças crônico-degenerativas, a partir do final do século XX, fez emergir no cenário nacional a figura do cuidador de idosos, evidenciando a importância de caracterizá-lo e de compreender suas necessidades. Esses idosos, dependentes de cuidadores, estão mais vulneráveis a violência, doenças, negligências e abandono (MINAYO MCS e GUALHANO L, 2018).

De acordo com a Classificação Brasileira de Ocupações do Ministério do Trabalho e Emprego o cuidador de idosos é uma profissão reconhecida, possui definição de Cuidador de Pessoas Idosas e Dependentes e Cuidador de Idosos Institucional, sendo pessoas que cuidam, mantendo bem-estar, saúde, alimentação, higiene pessoal, educação, cultura e recreação da pessoa em que estão cuidando, seguindo objetivos estabelecidos anteriormente (LAMPERT CDT, et al., 2016). O cuidador pode ser denominado de informal quando exerce esta atividade de forma não remunerada e de cuidador formal quando conta com o mínimo preparo e é remunerado pelo cuidado prestado (NARDI EDFR, et al., 2014).

À medida que aumentam a debilidade e a dependência do idoso, aumenta também a necessidade de um cuidador e, como resultado do ato de cuidar, pode gerar desgastes físicos e psicológicos para atender essas pessoas no desempenho de suas atividades diárias, que englobam desde a higiene pessoal até a oferta da medicação, com o propósito de melhorar a qualidade de vida dessas pessoas e, consequentemente, a capacidade funcional do idoso (SOUZA LR, et al., 2015). É considerado um indivíduo que absorve níveis diferentes de ansiedade, em função das características de sua atuação (VIEIRA ALT, et al., 2015).

As exigências da prestação de cuidados indicam um olhar para o cuidador, tendo em vista a necessidade de preservar o seu papel social e prevenir o risco de adoecer. O estresse advém da necessidade de cuidados ininterruptos, de lidar com situações difíceis, de manifestações psiquiátricas somadas a questões emocionais anteriores à instalação da doença (VIEIRA ALT, et al., 2015). Essa situação pode levar ao adoecimento do cuidador, bem como pode levar a situações de violências contra o idoso.

Nesse sentido, recente estudo transversal realizado em Manguinhos, na cidade do Rio de Janeiro com uma amostra de 135 duplas (idoso-cuidador) identificou que a presença de altos níveis de sobrecarga proporcionou um aumento de 11 vezes nas chances de ocorrência de violência de cuidadores contra idosos dependentes (LINO VTS, et al., 2019). 
Diante do quadro ora exposto, torna-se fundamental avaliar o nível de estresse dos cuidadores visando subsidiar estratégias de melhoria de sua qualidade de vida, sejam estas voltadas a ações de acompanhamento e assistência de sua saúde física e mental, sejam as de qualificações que busquem sanar dúvidas e minimizar as dificuldades encontradas no desenvolvimento de suas atividades diárias. Assim, o objetivo do presente estudo foi o de investigar o estresse dos cuidadores em Estratégias Saúde da Família (ESF) da zona rural e da zona urbana de um município do interior do Rio Grande do Sul.

\section{MÉTODOS}

Trata-se de uma pesquisa com abordagem qualitativa e descritiva (MINAYO MCS, 2010). A amostra foi composta por 10 cuidadores de idosos de quatro ESFs, sendo uma da zona rural e três da zona urbana, de um município situado na região noroeste do Estado do Rio Grande do Sul (Brasil). O critério de inclusão do estudo foi ser cuidador de algum idoso das áreas selecionadas, mesmo não tendo o registro ou qualificação formal comprovada para tal. Foram excluídas as pessoas com dificuldades de compreensão e/ou expressão verbal.

As ESFs da zona urbana foram escolhidas através de sorteio, pois o município conta com oito e somente uma na zona rural. $O$ acesso à lista de idosos ocorreu através do contato com as ESFs, somente após ser firmada a declaração da entidade coparticipante.

Para a coleta de dados foram utilizados um questionário, contendo informações relacionadas aos aspectos sociodemográficos e de saúde de cuidadores e idosos, e a Escala de Zarit, aplicada aos cuidadores, a qual é constituída por 22 itens, englobando avaliação do impacto percebido sobre a saúde física e emocional, atividades sociais e condições financeiras dos cuidadores. A escala de respostas varia de 0 a 4 , de acordo com a presença ou a intensidade de uma resposta afirmativa (nunca=0, raramente $=1$, algumas vezes $=2$, frequentemente $=3$ e sempre $=4$ ). A exceção é a última questão, no qual o entrevistado é abordado quanto a sobrecarga no papel de cuidador e as respostas podem ser: nem um pouco $=0$, um pouco $=1$, moderadamente $=2$, muito $=3$, extremamente $=4$ (BIANCHI M, et al., 2016).

A análise dos dados foi mediante análise de conteúdo temática das falas dos sujeitos (MINAYO MCS, 2010) e avaliação da Escala de Zarit. Foram mantidos o sigilo e o anonimato no que se refere à identificação dos participantes, bem como os outros aspectos éticos. Para isso, os participantes foram identificados com nome de pedras preciosas e aos ESFs foram atribuídos números de 1 a 5 , acrescido da letra $U$ para ESFs urbanas e R para a rural.

Os participantes que aceitaram fazer parte do estudo assinaram o Termo de Consentimento Livre e Esclarecido (TCLE), respeitando os aspectos Éticos e Legais vigentes, para a pesquisa envolvendo seres humanos, estabelecidas pela Resolução 466/2012 (BRASIL, 2012). A coleta de dados só teve início após parecer favorável do Comitê de Ética do Instituto Federal Farroupilha, sob o número do parecer 2.354.458. A fim de manter o sigilo e anonimato dos participantes, estes foram identificados pela letra $E$ de entrevistado, seguida do número arábico correspondente à ordem da coleta de dados (E1, E2, E3...E10).

\section{RESULTADOS E DISCUSSÃO}

A idade dos cuidadores variou de 26 a 78 anos, sendo dois do sexo masculino e oito femininos. Os cuidadores com mais idade são familiares. Nove idosos são da zona urbana, sendo sete do sexo feminino. Um do sexo feminino é da zona rural. A idade dos idosos variou de 70 a 90 anos, sendo cinco hipertensos e três hipertensos e diabéticos.

As dependências dos idosos, as quais se fizeram necessário cuidador foram: Doença de Alzheimer ( $n=03)$, Doença de Parkinson ( $n=01$ ), sequela de Acidente Vascular Encefálico (AVE) ( $n=01$ ), fraturas por quedas $(n=04)$, hipertensão, diabetes e depressão $(n=01)$. Cinco cuidadores são familiares do sexo feminino, um da zona rural e quatro da zona urbana. Os demais foram contratados através de uma "home care", que contrata os cuidadores que já tenham alguma formação na área da saúde, como técnicos em enfermagem ou estudantes de curso técnico e que já tenham habilidades como verificar pressão arterial, realizar curativo ou aplicar mediações, ou que tenham o curso de cuidador. 
Na presente pesquisa, dos cuidadores familiares, três possui mais de 60 anos e são esposas, e duas filhas (35 e 38 anos) e que não recebem remuneração pelo trabalho. O cuidador familiar assume esta responsabilidade por iniciativa ou determinação do grupo familiar, observando-se em geral, três aspectos: parentesco, gênero e proximidade física e afetiva (JESUS ITM, et al., 2018). Por ser natural acreditar que as mulheres devem assumir este papel de cuidar, muitas vezes, o estresse gerado por este trabalho não é valorizado e deixa de ser identificado por familiares.

$O$ estresse do cuidador pode ser definido como o efeito adverso que 0 ato de cuidar de alguém pode provocar no aspecto emocional, social, financeiro, físico e espiritual do cuidador (SOUZA LR, et al., 2015). O aumento das doenças crônicas e a longevidade da população contribuem para o número elevado de idosos com incapacidades que requerem cuidados específicos, com necessidade de cuidadores para estes indivíduos (MINAYO MCS e GUALHANO L, 2018).

Desse modo, o familiar nem sempre têm a opção de se tornar cuidador, pois acaba assumindo esse papel, muitas vezes, por não ter a possibilidade financeira para contratar alguém, assumindo a atividade que pode gerar situações de sobrecarga (OLIVEIRA JS, et al., 2018). Além disso, existe a realidade cultural de que a filha ou esposa devem assumir esse papel. No entanto, isso pode desencadear aspectos negativos ao familiar, como alterações no estado físico, emocional e desequilíbrio entre atividade e repouso (COUTO AM, et al., 2018).

O familiar, prestador de cuidado, aparece como um recurso para a desospitalização ou evitar a institucionalização. Entretanto, são pessoas sem preparação prévia, que realizam cuidados que seriam remunerados e de responsabilidade dos profissionais em meio hospitalar ou comunitário (COUTO AM, et al., 2018).

Os familiares cuidadores têm de garantir as tarefas que o idoso é dependente, tarefas como higiene pessoal e mobilidade. Além destes cuidados específicos destinados ao familiar, normalmente, eles precisam ainda realizar as tarefas diárias que já eram da sua responsabilidade anteriormente: cozinha, faxina, cuidados com os filhos, entre outras tarefas, simultaneamente (COUTO AM, et al., 2018), como relatam os entrevistados:

\footnotetext{
“...muitas vezes estou muito cansada e estressada, pois preciso cozinhar para meus filhos, limpar a casa, lavar roupa e ainda preciso estudar pras provas, pois voltei a estudar e tenho que cuidar e dar atenção pro pai." $\left(\mathrm{E}_{2}\right)$

"...chego em casa cansada do trabalho do dia e da aula de noite, e quando chego minha mãe quer atenção, quer ir ao banheiro, quer comer... Isso me esgota...." (E $\left.\mathrm{E}_{4}\right)$

“...ele não é fácil de cuidar. Tem que ser como e quando ele quer.” ( $\left.E_{5}\right)$
}

O predomínio de cuidadores do sexo feminino corrobora com outros estudos que reforçam o papel social da mulher, que historicamente é determinado com a função de provedora de cuidados. Nesse sentido, 0 cuidado ao familiar idoso é somente mais uma tarefa que a mulher assume no âmbito doméstico (ANJOS KF, et al., 2014), impactando diretamente na qualidade de vida desses cuidadores.

Alguns países latinos têm a intenção de diminuir gastos com o cuidado para portadores de doenças crônicas ou pessoas dependentes de cuidados paliativos, que dependem de um cuidador cotidiano de responsabilidade da família. Para isso, o Estado precisa transferir os custos do cuidado para as famílias como a produção de significativas alterações na organização e nos modos de vida das famílias, o que implica em renunciar ao trabalho remunerado (SIMÃO VM e MIOTO RCT, 2016).

No Brasil, a Lei no 10.741 , de 1ํ de outubro de 2003, que dispõe sobre o Estatuto do Idoso e dá outras providências, expressa em seu artigo terceiro a obrigação da família, da comunidade, da sociedade e do poder público assegurar ao idoso, com absoluta prioridade, a efetivação dos seus direitos (BRASIL, 2003). Porém, para esta efetivação, torna-se fundamental a estruturação dos serviços públicos na área da saúde, da assistência social e dos direitos humanos para que sejam supridas as necessidades de idosos vulneráveis, 
dependentes ou em situação de fragilidade a fim de que novas formas de articulação em rede, venham a oferecer o cuidador formal para as famílias que dele necessitarem, estruturação essa que, em consonância com o observado no presente estudo, ainda não fora implementada.

Nesse sentido, os profissionais que integram as equipes das ESFs reconhecem a importância do cuidador ou familiar do idoso na concretização da assistência a pessoa idosa no domicílio. Dessa forma, devem orientar esses cuidadores a fim de que esse cuidado possa de dar de forma compartilhada, em que várias pessoas podem se responsabilizar pelo tratamento, não havendo, assim, sobrecarga para ninguém.

Nesse contexto, é importante reforçar o papel das equipes da ESF no treinamento e na supervisão dos cuidadores, pois o profissional somente vai conquistar a autonomia do cuidador quando este for incorporado ao processo de trabalho das equipes, considerando as questões ético-legais existentes no cuidado domiciliar. As atividades desenvolvidas pelos cuidadores informais em domicílio, em algumas situações, são de alta complexidade e realizadas sem a supervisão de um profissional com competência legal, o que acarreta prejuízo no cuidado ao paciente e até situações de risco (MUNIZ EA, et al., 2016).

Assim, a partir do momento em que eles perceberem que não estão sozinhos para prestar estes cuidados, poderão requerer auxílio quando necessitarem. Ressalta-se que o cuidado em ambiente domiciliar permite $o$ aumento da qualidade do cuidado, contribuindo para a redução da demanda por atendimentos hospitalares, o tempo médio de permanência na internação, os riscos de infecção hospitalar com ênfase para as infecções cruzadas e os custos associados a estas situações (VASCONCELOS GB e PEREIRA PM, 2018).

$\mathrm{Na}$ avaliação da sobrecarga dos cuidadores familiares, através da Escala de Zarit (BIANCHI M, et al., 2016), um dos cuidadores apresentou sobrecarga moderada a severa e os demais sobrecarga moderada. As atribuições devem ser pactuadas entre equipe, família e cuidador, democratizando saberes, poderes e responsabilidades (SIMÃO VM e MIOTO RCT, 2016).

No entanto, o cuidador, muitas vezes, não aceita ajuda e acha que somente ele conhece a maneira de prestar os cuidados, gerando certo cansaço nesse cuidador, que pode apresentar quadros depressivos e, com isso, a diminuição da qualidade da assistência prestada. Em um estudo semelhante, 10\% dos 322 idosos usuários da atenção primária de Niterói (Rio de Janeiro) relataram ter sofrido violência física por parte de familiares (APRATTO JUNIOR PC, 2010).

Nesta perspectiva, a ESF ou a Unidade Básica de Saúde (UBS) têm o papel de fornecer orientações ao cuidador da pessoa idosa, conquistando a sua confiança e seu respeito. A equipe deve, após orientar o cuidador, realizar avaliações em que serão determinadas as necessidades dos cuidadores e da pessoa idosa (FLORIANO LA, et al., 2012).

A cooperação a ser estabelecida entre os profissionais de saúde das ESF ou UBS e os cuidadores, sejam eles formais ou informais, deverá assegurar a sistematização das tarefas a serem realizadas no próprio domicílio da pessoa idosa direcionadas à esta, bem como, ao seu cuidador.

Entre as atividades a serem consideradas estão as relacionadas à promoção da saúde, à prevenção de incapacidades, à manutenção da capacidade funcional e autonomia com isso evitando, na medida do possível, hospitalização, institucionalização e/ou outras formas de segregação e isolamento (BRITO MCC, et al., 2013).

No presente estudo se percebeu nas ESFs a fragilidade das ações de apoio ou suporte às famílias que possuem cuidador familiar e desenvolvem essa atividade sem treinamento, utilizando apenas um conhecimento empírico, de forma solitária e desgastante. Nesse sentido, urge a necessidade de sensibilização dos gestores e profissionais de saúde sobre a importância do cuidado domiciliar ao idoso e ao cuidador, promovendo estratégias de apoio e capacitação para os cuidadores.

A presença do cuidador no domicílio na época atual é de suma importância, pois contribui para diminuir as internações hospitalares, institucionalizações ou isolamento do idoso. No entanto, percebe-se que a saúde destes, em alguns casos, está comprometida, o que faz supor estar aumentada a probabilidade de riscos na execução de suas tarefas (GUTIERREZ LLP, et al., 2017). 
Na escala de avaliação da sobrecarga dos cuidadores, a Escala de Zarit, no item 19, refere-se às dúvidas sobre o que fazer pelo idoso. Nesse sentido, dois entrevistados responderam que sempre tem dúvidas, outros dois afirmaram que às vezes e um deles disse que frequentemente. Isso representa $50 \%$ dos entrevistados, apesar de que não faz parte da rotina do cuidador familiar técnicas e procedimentos inerentes a área de enfermagem:

\author{
“...acho que poderia cuidar melhor do idoso." (E7) \\ “...as vezes tenho dúvidas no que é melhor fazer em determinadas situações...” ( $\left.\mathrm{E}_{8}\right)$
}

O cuidador do idoso deveria ser uma pessoa capacitada e que fizesse o elo entre o idoso e a família, o serviço de saúde e a comunidade (MIRANDA GMD, et al., 2016). Isso possibilita uma reflexão de forma consciente sobre as ações e atitudes destes cuidadores. Sabe-se que, muitas vezes, esse cuidador não tem toda a segurança no sentido de possibilitar o desenvolvimento de práticas holísticas, reflexivas e capazes de subsidiar a assistência do cuidador.

$\mathrm{Na}$ avaliação da sobrecarga dos cuidadores contratados, através da escala, um dos cuidadores apresentou sobrecarga moderada a severa e os demais sobrecarga moderada, como foi o caso dos cuidadores familiares. A sobrecarga constitui-se em fator de risco para violência realizada por cuidadores de idosos dementes e conjuntamente daqueles dependentes para as atividades de vida diária (BIANCHI M, et al., 2016).

Estes cuidadores são expostos a diversos fatores que podem causar sofrimento, como a alta demanda física e sobrecarga emocional em conviver com o doente. Além disso, a falta de conhecimento suficiente para desenvolver um bom cuidado, privação de sono para trabalhar como cuidador e outras atividades cotidianas acabam provocando o estresse. Os cinco cuidadores contratados revelaram que gastam bastante tempo com o idoso, restando pouco tempo para cuidar de si próprio:

"...fico oito horas durante o dia com o idoso. Tenho que trocar a fralda, fazer mudança de decúbito, dar medicação e alimentação, retirar do leito, dar banho...e aí não tenho tempo para estudar. Mas fazer o que? Preciso trabalhar. É desgastante." $\left(\mathrm{E}_{2}\right)$

À medida que aumentam a debilidade e a dependência do idoso, o cuidar sofre mudanças, pois passa a exigir mais do cuidador para suprir as necessidades geradas pela diminuição da capacidade funcional. Quanto à saúde do cuidador, pode provocar ansiedade, estresse ou depressão e, nesses casos, o cuidador pode deixar de lado as atividades de lazer e o autocuidado, ocasionando prejuízos na qualidade de vida e no cuidado prestado ao idoso (SOUZA LR, et al., 2015).

\title{
CONCLUSÃO
}

Devido a fatores socioeconômicos das famílias, principalmente no Brasil, o cuidador de um idoso dependente normalmente é um familiar, do sexo feminino e é o responsável pelo processo de cuidar e reabilitar no próprio domicílio, o que desencadeia estresse e desgaste, provocando fatores de risco à saúde desse cuidador devido a sobrecarga de atividades cotidianas referentes ao cuidar e ao próprio idoso exposto a possíveis situações de violência. A sobrecarga deve ser vista como um conceito multidimensional, abrangendo muitas variáveis como tempo disponível para o cuidado, recursos financeiros, condições psicológicas, físicas e sociais, atribuições e distribuição de papéis. É necessária uma rede de atenção à saúde do idoso para ofertar diversos tipos de serviços, com apoio financeiro e estruturada de forma regionalizada e integrada. Nesse sentido, as ESFs se apresentam como uma alternativa de suporte formal para o idoso e a família, visto que entre as atividades que devem ser exercidas pelos profissionais das equipes está a educação em saúde, principalmente, relacionada a execução do cuidado e a adoção de medidas preventivas de incapacidades e doenças, propiciando autonomia. 


\section{REFERÊNCIAS}

1. ANJOS KF, et al. Qualidade de vida de cuidadores familiares de idosos dependentes no domicílio. Texto Contexto Enferm, 2014; 23(3): 600-8.

2. APRATTO JUNIOR PC. A violência doméstica contra idosos nas áreas de abrangência do Programa Saúde da Família de Niterói (RJ, Brasil). Ciênc. saúde coletiva, 2010; 15 (6): 2983-95.

3. BIANCHI M, et al. Indicadores psicométricos da Zarit Burden Interview aplicada a idosos cuidadores de outros idosos. Rev. Latino-Am. Enfermagem, 2016; 24: e2835.

4. BRASIL. Lei n. 10.741, de 1ํ de outubro de 2003. Dispõe sobre o Estatuto do Idoso e dá outras providências. Brasília, DF, 2003.

5. BRASIL. Ministério da Saúde. Conselho Nacional de Saúde. Resolução n. 466, de 12 de dezembro de 2012. Aprova diretrizes e normas regulamentadoras de pesquisas envolvendo seres humanos. Brasília, Diário Oficial da União, 2012.

6. BRASIL.Instituto Brasileiro de Geografia e Estatística. Tábua completa de mortalidade para o Brasil - 2018: breve análise da evolução da mortalidade no Brasil. Rio de Janeiro, 2019.

7. BRITO MCC, et al. Envelhecimento populacional e os desafios para a saúde pública: análise da produção científica. Revista Kairós Gerontologia, 2013; 16(3): 161-178.

8. COUTO AM, et al. Cuidador familiar de idosos e o cuidado cultural na assistência de enfermagem. Rev Bras Enferm., 2018; 71(3): 1020-8.

9. FLORIANO LA, et al. Cuidado realizado pelo cuidador familiar ao idoso dependente, e domicílio, no contexto da Estratégia de Saúde da Família. Texto Contexto Enferm, 2012; 21(3): 543-8.

10. GUTIERREZ LLP, et al. Caracterização de cuidadores de idosos da região metropolitana de Porto Alegre (RS): perfil do cuidado. Saúde Debate, 2017; 41(114): 885-898.

11. JESUS ITM, et al. Sobrecarga, perfil e cuidado: cuidadores de idosos em vulnerabilidade social.Rev. Bras. Geriatr. Gerontol, 2018; 21(2): 194-204.

12. LAMPERT CDT, et al. Dispositivos legais no trabalho de cuidadores: aplicação em Instituições de Longa Permanência. REAd. Rev. eletrôn. adm. (Porto Alegre), 2016; 22(3): 360-380.

13. LINO VTS, et al. Prevalência e fatores associados ao abuso de cuidadores contra idosos dependentes: a face oculta da violência familiar.Ciênc. saúde coletiva, Rio de Janeiro, 2019; 24 (1): 87-96.

14. MINAYO MCS. O desafio do conhecimento. 12. ed. São Paulo, 2010.

15. MINAYO MCS, GUALHANO L. Problemas de saúde e vulnerabilidade da população idosa. SciELO em Perspectiva, 2018.

16. MIRANDA GMD, et al. O envelhecimento populacional brasileiro: desafios e consequências sociais atuais e futuras. Rev. Bras. Geriatr. Gerontol., 2016; 19(3): 507-519.

17. MUNIZ EA, et al. Grau de sobrecarga dos cuidadores de idosos atendidos em domicílio pela Estratégia Saúde da Família. Saúde debate, 2016; 40(110).

18. NARDI EDFR, et al. Dificuldades dos cuidadores familiares no cuidar de um idoso dependente no domicílio. Ciênc Cuid Saúde. 2012;11(1):98-105

19. OLIVEIRA JS, et al. Repercussões na vida de cuidadores de crianças e adolescentes com doença oncológica. Cogitare Enferm., 2018; (23)2: e51589.

20. SIMÃO VM, MIOTO RCT. O cuidado paliativo e domiciliar em países da América Latina. Saúde em Debate, 2016; 40(108): 156-169.

21. SOUZA LR, et al. Sobrecarga no cuidado, estresse e impacto na qualidade de vida de cuidadores domiciliares assistidos na atenção básica. Cad. Saúde Colet., 2015; 23(2): 140-149.

22. VASCONCELOS GB, PEREIRA PM. Cuidados paliativos em atenção domiciliar: uma revisão bibliográfica. Rev. Adm. Saúde, 2018; 18(70).

23. VIEIRA ALT, et al. Avaliação do estresse em cuidadores de idosos na Unidade de Saúde da Família "Vila Saúde". M \& P Medicina \& Pesquisa, 2015; 1(1): 61-74.

24. ZAMBONI C, et al. Violência contra idoso: um velho estigma. Cogitare Enferm., 2011; 16 (4): 634-9. 